VOL. 8 (1973), 247-259

\title{
On a class of diophantine inequalities
}

\section{Kurt Mahler}

Dedicated to B. Segre, on his 70th birthday, 16 February 1973.

As a special case of more general results, it is proved in this note that, if $\alpha$ is any real number and $\delta$ any positive number, then there exists a positive integer $X$ such that the inequality

$$
\left|X\left(\frac{3}{2}\right)^{h}-y_{h}-\alpha\right|<\delta
$$

has infinitely many solutions in positive integers $h$ and $y_{h}$. The method depends on the study of infinite sequences of real linear forms in a fixed number of variables. It has relations to that used by Kronecker in the proof of his classical theorem and can be generalised.

For real a put

$$
\|\alpha\|=\min _{y=0, \pm 1, \pm 2, \ldots}|\alpha-y|
$$

so that $\|\alpha\|$ denotes the distance of $\alpha$ from the nearest integer and hence that

$$
0 \leq\|\alpha\| \leq \frac{1}{2}
$$

By $H_{0}$ we understand a fixed strictly increasing infinite sequence of positive integers $h$ ( $B_{0}$ usually will be the set of all positive Received 20 November 1972. 
integers), and $B$ denotes some infinite subsequence of $B_{0}$, not necessarily always the same.

2.

Let $r$ be a fixed and $n$ a variable positive integer; let further $s_{n}$ be the set of all $n$-vectors $\mathrm{x}=\left(x_{1}, \ldots, x_{p}\right)$ with integral components satisfying

$$
1 \leq \max \left(\left|x_{1}\right|, \ldots,\left|x_{p}\right|\right) \leq n .
$$

Thus $S_{n}$ is a finite set, and all vectors in $S_{n}$ are distinct from the zero vector

$$
0=(0, \ldots, 0) .
$$

Next consider an infinite sequence of $p$-vectors

$$
a_{h}=\left(a_{h l}, \ldots, a_{h r}\right) \quad\left(h \in H_{0}\right)
$$

with real components and the associated linear forms

$$
L_{h}(\mathrm{x})=a_{h 1_{1}} x_{1}+\ldots+a_{h r_{r}} \quad\left(h \in H_{0}\right)
$$

in $x$. Then put

$$
M_{h}(n)=\min _{x \in S_{n}}\left\|L_{h}(x)\right\| \quad\left(h \in H_{0}\right)
$$

and

It is obvious that

$$
0 \leq M_{h}(n) \leq \frac{1}{2} \quad\left(h \in H_{0}\right)
$$

and hence that also

$$
\text { Q } \leq M(n) \leq \frac{1}{2} \text {. }
$$

3.

For $n \geq 3$ these upper bounds for $M_{h}(n)$ and $M(n)$ can be improved. 
For this purpose, denote by $y$ a further integral variable. The system of $r+1$ linear forms

$$
n^{-1} x_{1}, \ldots, n^{-1} x_{r}, n^{r}\left(a_{h 1} x_{1}+\ldots+a_{h r^{x}} x^{-y}\right) \quad\left(h \in H_{0}\right)
$$

in $x_{1}, \ldots, x_{r}, y$ has the determinant -1 . Hence, by Minkowski's Theorem on linear forms, there exist integers

$$
x_{h l}, \ldots, x_{h r}, y_{h}
$$

not all zero, which in general will depend on $h$, such that simultaneously $\max \left(\left|x_{h l}\right|, \ldots,\left|x_{h r}\right|\right) \leq n,\left|a_{h l} x_{h I}+\ldots+a_{h r} x_{h r}-y_{h}\right|<n^{-r} \quad\left(h \in H_{0}\right)$.

Here at least one of the first $r$ integers

$$
x_{h l}, \ldots, x_{h r}
$$

does not vanish. For otherwise $y_{h} \neq 0$, whence

$$
1 \leq\left|y_{h}\right|<n^{-r} \leq 1
$$

which is impossible.

The vector

$$
x_{h}=\left(x_{h 1}, \ldots, x_{h r}\right)
$$

therefore lies in $S_{n}$ and in addition satisfies the inequality

$$
\left\|L_{h}\left(x_{h}\right)\right\|<n^{-r} \quad\left(h \in H_{0}\right)
$$

From this it follows immediately that

$$
0 \leq M_{h}(n)<n^{-r} \quad\left(h \in H_{0}\right)
$$

and hence also that

$$
0 \leq M(n) \leq n^{-r} \text {. }
$$

On the other hand, since obviously $S_{n} \subset S_{n+1}$, it is clear that

$$
M_{h}(1) \geq M_{h}(2) \geq M_{h}(3) \geq \ldots \geq 0 \quad\left(h \in H_{0}\right) \text {, }
$$


from which it is easily deduced that also

$$
M(1) \geq M(2) \geq M(3) \geq \ldots \geq 0 \text {. }
$$

4.

The definition of $M(n)$ as an upper limit implies that there exists a subsequence $H$ of $H_{0}$ such that

$$
\lim _{\substack{h \rightarrow \infty \\ h \in H}} M_{h}(n)=M(n) .
$$

Here, to each suffix $h$ in $H$, we can find a vector $x_{h}$ in $S_{n}$ such that

$$
M_{h}(n)=\left\|L_{h}\left(x_{h}\right)\right\| \quad(h \in H) ;
$$

note that $x_{h}$ need not be the same as the vestor $x_{h}$ constructed in $\S 3$.

As $h$ runs over $H, x_{h}$ is restricted by the condition of belonging to the finite set $S_{n}$. Therefore, if necessary, $H$ can be replaced by an infinite subsequence which we call again $H$ such that, without loss of generality,

$$
x=x_{h} \text { for all } h \in H
$$

is a fixed vector in $S_{n}$ independent of $h$; naturally,

$$
x \neq 0 \text {. }
$$

Since this vector has the basic property that

$$
\lim _{\substack{h \rightarrow \infty \\ h \in H}}\left\|L_{h}(x)\right\|=M(n),
$$

the following result has been established.

LEMMA 1. For every positive integer $n$ there exist an infinite subsequence $H$ of $H_{0}$ and a constant vector $x$ in $S_{n}$ with the property (3). 


\section{5.}

In this lemma, $H$ will in general be a proper subsequence of $H_{0}$ as the following example shows.

Fix $n$ and choose $r=1$ so that $a_{h}$ and $x$ are now scalars $a_{h}$ and $x$. As the linear forms take

$$
L_{h}(x)= \begin{cases}x & \text { if } h \text { is even, } \\ x \sqrt{2} & \text { if } h \text { is odd. }\end{cases}
$$

In this example, $M_{h}(n)$ evidently vanishes for even $h$ (we may put $x=1$ ), but is positive and independent of $h$ for odd $h$. Hence also $M(n)$ is positive. Thus, if $H_{0}$ is the set of all positive integers $h, H$ in (3) essentially (that is, except for possibly finitely many even numbers) is the sequence of all odd integers.

6.

Consider again the general case, but assume that, for a certain $n$, $M(n)=0$. Since $M_{h}(n) \geq 0$ for all $h \in H_{0}$, it is clear that now the upper limit in the definition of $M(n)$ becomes the limit, hence that (3) takes the form

$$
\lim _{\substack{h \rightarrow \infty \\ h \in H_{0}}}\left\|L_{h}(x)\right\|=0 .
$$

Denote by $\alpha$ an arbitrary real number which is not an integer. The relation (4) implies that

$$
\lim _{\substack{h \rightarrow \infty \\ h \in H_{0}}}\left\|L_{h}(x)-\alpha\right\|=\|\alpha\|>0 .
$$

This formula suggests the problem whether there exist an infinite subsequence $B$ of $H_{0}$ and an integral vector $X$ distinct from $X$ such that

$$
\lim _{\substack{h \rightarrow \infty \\ h \in H}}\left\|L_{h}(X)-\alpha\right\|=0 .
$$


The answer to this problem depends very much on the special forms $L_{h}$ and the sequences $H_{0}$ and $H$.

A positive answer can be given in the following trivial example. Let $r=1$ and $n=2$; let $H_{0}$ and $H$ be the sequences of all positive integers and of all odd positive integers, respectively; and let further

$$
L_{h}(x)=\frac{1}{2} x \text { for } h \in H_{0} \text {. }
$$

Since $L_{h}(2)=1$, evidently

$$
M_{h}(2)=M(2)=0 \text {. }
$$

On the other hand,

$$
\left\|L_{h}(1)-\frac{1}{2}\right\|=0 \text { for all } h \in H \text {. }
$$

A negative answer holds in the following rather more interesting exámple. Let again $r=1$, and let $H_{0}$ be again the sequence of all positive integers. Assume that the forms $L_{h}$ heve the property

$$
\lim _{\substack{h \rightarrow \infty \\ h \in H_{0}}}\left\|L_{h}(1)\right\|=0 .
$$

Then obviously also

$$
\lim _{\substack{h \rightarrow \infty \\ h \in H_{0}}}\left\|L_{h}(x)\right\|=0 \text { for every integer } x,
$$

and hence there cannot exist a subsequence $H$ of $H_{0}$ and an integer $X$ satis fying

$$
\lim _{\substack{h \rightarrow \infty \\ h \in H}}\left\|L_{h}(X)-\alpha\right\|=0
$$

unless $\alpha$ is an integer.

A simple example in which the condition (5) is satisfied and therefore also the conclusion about (7) is given by the linear forms 


$$
L_{h}(x)=h ! e x \text { for } h \in H_{0}
$$

where $H_{0}$ still denotes the sequence of all positive integers.

of much greater interest is, however, the sequence of forms

$$
L_{h}(x)=\lambda \theta^{n} x \text { for } h \in H_{0}
$$

where $\theta>1$ is a fixed algebraic number, and $\lambda>0$ is a constant. A theorem due to Pisot [1] (see also Salem [2]) asserts that the limit equation

$$
\lim _{h \rightarrow \infty}\left\|\lambda \theta^{h}\right\|=0
$$

that is, the condition (5), is satisfied if and only if the following two properties hold.

(i) $\theta=\theta^{(1)}$ is an algebraic integer of some degree $m \geq 1$ such that all its algebraic conjugates $\theta^{(2)}, \ldots, \theta^{(m)}$ are less than 1 in absolute value.

(ii) $\lambda$ lies in the algebraic number field $Q(\theta)$ generated by $\theta$. Call $\{\theta, \lambda\}$ a Pisot pair whenever these two properties are satisfied. By (7), such pairs have the following further property.

(iii) If $\alpha$ is any real number, $H$ any subsequence of $H_{0}$, and $X$ any integer, then the equation

$$
\begin{aligned}
& \lim _{\substack{h \rightarrow \infty \\
h \in H}}\left\|\lambda \theta^{h} X-\alpha\right\|=0 \\
&
\end{aligned}
$$

implies that $\alpha$ is an integer.

If $\{\theta, \lambda\}$ is a Pisot pair, then by (6) the forms (8) satisfy

$$
M(n)=0 \text { for all } n \geq 1 \text {. }
$$

This result has a converse. For as sume that $\{\theta, \lambda\}$ is not necessarily a Pisot pair, but that (9) is true. This equation (9) is equivalent to 


$$
\lim _{h \rightarrow \infty} \min _{h \in H_{0}}\left\|\lambda \theta^{h} x\right\|=0 .
$$

Now for every real number $\alpha$ and for every integer $g$,

$$
\|g \alpha\| \leq|g| \cdot\|\alpha\|
$$

hence

$$
\left\|n ! \lambda \theta^{h}\right\| \leq n ! \min _{x= \pm 1, \pm 2, \ldots, \pm n}\left\|\lambda \theta^{h} x\right\|
$$

because all factors $x$ are divisors of $n !$. The equation (10) implies then that

$$
\lim _{h \rightarrow \infty}\left\|n ! \lambda \theta^{h}\right\|=0
$$

This, however, means that $\{\theta, n ! \lambda\}$ and hence also $\{\theta, \lambda\}$. are Pisot pairs. Thus the following result holds.

LEMMA 2. Let $\theta>1$ be an algebraic number and $\lambda$ a positive number, let again $H_{0}$ be the sequence of all positive integers, and Let

$$
L_{h}(x)=\lambda \theta^{h} x \text { for } h \in H_{0} \text {. }
$$

Then $\{\theta, \lambda\}$ is a Pisot pair if and only if

$$
M(n)=0 \text { for all } n \geq 1 \text {. }
$$

8.

We return to the general case of $\$ 2$, but assume now that for a certain value of $n$,

$$
M(n)>0
$$

Denote by $x$ the constant vector in $S_{n}$ given by Lemma 1 and for which

$$
\lim _{\substack{h \rightarrow \infty \\ h \in H}}\left\|L_{h}(x)\right\|=M(n) .
$$

It follows that there exists an infinite subsequence of $H$ which we call 
again $H$ such that

$$
\frac{2}{3} M(n)<\left\|L_{h}(x)\right\|<\frac{4}{3} M(n) \text { for all } h \in H
$$

In explicit form, $x=\left(x_{1}, \ldots, x_{p}\right)$, and there exists to each $h \in H$ an integer $y_{h}$ such that the sum

$$
s_{h}=a_{h 1} x_{1}+\ldots+a_{h r} x_{r}-y_{h}
$$

satisfies the equation

$$
\left|s_{h}\right|=\left\|L_{h}(x)\right\|
$$

and therefore also the inequality

$$
\frac{2}{3} M(n)<\left|s_{h}\right|<\frac{4}{3} M(n) \text { for all } h \in H .
$$

9.

Next let $\alpha$ be an arbitrary real number, and let $y$ be the unique integer for which the real number

$$
\beta=\alpha+y
$$

satisfies the inequality

$$
\frac{2}{3}<B \leq \frac{5}{3} \text {. }
$$

The integral multiples

$$
s_{h} s \quad(z=0, \pm 1, \pm 2, \ldots)
$$

of $s_{h}$ form an arithmetic progression of distance $\left|s_{h}\right|>0$. By (II), every open interval of length $\frac{4}{3} M(n)$ contains then at least one element of this progression.

We apply this property to the open interval

$$
\text { from } \beta-\frac{2}{3} M(n) \text { to } \beta+\frac{2}{3} M(n)
$$

of this length and deduce that

for every $h \in H$ there exists an integer $z_{h}$ such that

$$
-\frac{2}{3} M(n)<s_{h} z h-B<\frac{2}{3} M(n) \text {. }
$$


Here $\beta \leq \frac{5}{3}$ and $M(n) \leq \frac{1}{2}$, so that by (11),

$$
\left|z_{h}\right|<\frac{\beta+\frac{2}{3} M(n)}{\frac{2}{3} M(n)} \leq \frac{5+2 M(n)}{2 M(n)}
$$

and therefore

$$
\left|z_{h}\right|<\frac{3}{M(n)} .
$$

On the other hand, $\beta>\frac{2}{3}$, and so again by (ll),

$$
s_{h}{ }_{h}>\beta-\frac{2}{3} M(n) \geq \frac{2}{3}-\frac{1}{3}>0 \text {, }
$$

whence also

$$
z_{h} \neq 0
$$

In this construction, $z_{h}$ is a function of $h \in H$ which, by (13), has only finitely many possible values. Since $H$ may, if necessary, once more be replaced by a suitable infinite subsequence, we may without loss of generality assume that

$$
z_{h}=z \text { for all } h \in H
$$

has a fixed integral value independent of $h$, where by (13) and (14)

$$
0<|z|<\frac{3}{M(n)}
$$

10.

Put finally

$$
X_{1}=x_{1} z, \ldots, X_{r}=x_{r} z, y_{h}=y_{h} z+y .
$$

Then $\mathrm{X}=\left(x_{1}, \ldots, x_{r}\right)$ is an integral $p$-vector independent of $h$ such that

$$
1 \leq \max \left(\left|x_{1}\right|, \ldots,\left|x_{p}\right|\right)<\frac{3 n}{M(n)}
$$

while $y_{h}$ is an integer which in general depends on $h$. In this new notation, the lower and upper estimates for ${ }^{s_{h}} z_{h}-\beta$ take the form 


$$
-\frac{2}{3} M(n)<L_{h}(X)-Y_{h}-\alpha<\frac{2}{3} M(n) \text { for all } h \in H \text {. }
$$

Since $\frac{2}{3} M(n)<\frac{1}{2}$, this is equivalent to

$$
\left\|L_{h}(X)-\alpha\right\|<\frac{2}{3} M(n) \text { for all } h \in H \text {. }
$$

Thus the following result has been obtained.

LEMMA 3. For a certain $n \geq 1$ let $M(n)>0$. Then, to every real number $\alpha$, there exist an infinite subsequence $H$ of $H_{0}$ and a constant integral vector $X$ such that both (16) and (17) are satisfied.

This lemma becomes particularly interesting when $M(n)$ is positive for all. positive integers $n$. For, by the earlier estimate (2),

$$
\lim _{n \rightarrow \infty} M(n)=0 \text {. }
$$

Therefore, for sufficiently large $n$, the right-hand side of (17) can be made arbitrarily small, giving the following result.

THEOREM 1. Let $r \geq 1$ be a fixed integer, and let $H_{0}$ be a strictly increasing infinite sequence of positive integers. Associate with each $h$ in $H_{0}$ a real linear form

$$
L_{h}(x)=a_{h 1} x_{1}+\ldots+a_{h r_{r}},
$$

and assume that the upper limit $M(n)$, as defined in $\$ 2$, is positive for every positive integer $n$.

Then, given cony real number $\alpha$ and any positive number $\delta$, there exist an infinite subsequence $H$ of $H_{0}$ and an integral vector $X \neq 0$ independent of $h$ such that

$$
\left\|L_{h}(X)-\alpha\right\|<\delta \text { for alz suffices } h \text { in } H \text {. }
$$

11.

We combine this theorem with Lemma 2, taking $r=1$. Let $\theta$ and $\lambda$ be as in Lemma 2, but assume that $\{\theta, \lambda\}$ is not a Pisot pair. Then $M(n)$ is positive for all $n \geq 1$, and Theorem 1 gives the following consequence.

THEOREM 2. Let $\theta>1$ be con algebraic number, and $\lambda>0$ a 
constant. Assume that at least one of the following two properties is not satisfied.

(i) $\theta=\theta^{(1)}$ is an algebraic integer of degree $m \geq 1$ such that all its algebraic conjugates $\theta^{(2)}, \ldots, \theta^{(m)}$ have absolute values less thon 1 .

(ii) $\lambda$ lies in the algebraic number field $Q(\theta)$ generated by $\theta$. Then, given any real number $\alpha$ and any positive number $\delta$, there exists $a$ positive integer $X$ such that the inequality

$$
\left\|x \lambda \theta^{h}-\alpha\right\|<\delta
$$

has infinitely many solutions in positive integers $h$.

By way of example, this theorem can be applied to each of the inequalities

$$
\left\|x \sqrt{2}\left(\frac{1+\sqrt{5}}{2}\right)^{h}-\alpha\right\|<\delta,\left\|X e(1+\sqrt{2})^{h}-\alpha\right\|<\delta,\left\|X \lambda\left(\frac{3}{2}\right)^{h}-\alpha\right\|<\delta,
$$

where in the last inequality $\lambda$ may be an arbitrary positive number.

12.

We conclude this note with an application of Theorem 1 when $r$ is an arbitrary positive integer. For this purpose, assume that

$$
L_{h}(x)=a_{1} x_{1}+\ldots+a_{r^{x}} x_{r}
$$

does not depend on $h$. Any relation $M(n)=0$ where $n \geq 1$ now implies that the numbers

$$
a_{1}, \ldots, a_{r}, 1
$$

are linearly dependent over the rational field $Q$. Conversely, if these numbers are linearly independent over $Q$, then $M(n)$ is positive for all $n>1$. In this case it follows from Theorem 1 that for every real number $\alpha$ and for every positive number $\delta$ there exist $r$ integers $X_{1}, \ldots, X_{r}$ not all zero such that

$$
\left\|a_{1} X_{1}+\ldots+a_{r} X_{r}-\alpha\right\|<\delta .
$$


We obtain thus a rather special case of Kronecker's Theorem.

\section{References}

[1] Charles Pisot, "La repartition modulo 1 et les nombres algébriques", Ann. Scuola Norm. Sup. Pisa, Sci. Fis. Math. (2) 7 (1938), 205-248.

[2] Raphael Salem, Algebraic numbers and Fourier canalysis (D.C. Heath and Co., Boston, Massachusetts, 1963).

Department of Mathematics, Institute of Advanced Studies, Australian National University, Canberra, ACT. 